\title{
Wavelength Conversion with Large Signal-Idler Separation using Discrete Four-Wave Mixing in a Silicon Nanowire
}

Hu, Hao; Peucheret, Christophe; Pu, Minhao; Ji, Hua; Galili, Michael; Yvind, Kresten; Hvam, Jørn Märcher; Jeppesen, Palle; Oxenløwe, Leif Katsuo

\author{
Published in: \\ CLEO Technical Digest
}

Publication date:

2012

Document Version

Publisher's PDF, also known as Version of record

Link back to DTU Orbit

Citation (APA):

Hu, H., Peucheret, C., Pu, M., Ji, H., Galili, M., Yvind, K., Hvam, J. M., Jeppesen, P., \& Oxenløwe, L. K. (2012). Wavelength Conversion with Large Signal-Idler Separation using Discrete Four-Wave Mixing in a Silicon Nanowire. In CLEO Technical Digest (pp. CW1A.2). Optical Society of America.

\section{General rights}

Copyright and moral rights for the publications made accessible in the public portal are retained by the authors and/or other copyright owners and it is a condition of accessing publications that users recognise and abide by the legal requirements associated with these rights.

- Users may download and print one copy of any publication from the public portal for the purpose of private study or research.

- You may not further distribute the material or use it for any profit-making activity or commercial gain

- You may freely distribute the URL identifying the publication in the public portal 


\title{
Wavelength Conversion with Large Signal-Idler Separation using Discrete Four-Wave Mixing in a Silicon Nanowire
}

\author{
Hao Hu, Christophe Peucheret, Minhao Pu, Hua Ji, Michael Galili, Kresten Yvind, Jørn M. Hvam, \\ Palle Jeppesen, and Leif K. Oxenløwe \\ DTU Fotonik, Technical University of Denmark, Building 343, DK-2800 Lyngby, Denmark \\ huhao@fotonik.dtu.dk
}

\begin{abstract}
We have demonstrated wavelength conversion over $468 \mathrm{~nm}$ based on discrete bands phase matching in a silicon nanowire. CW light is converted from $1258 \mathrm{~nm}$ to $1726 \mathrm{~nm}$ with a CW pump at $1455 \mathrm{~nm}$.

OCIS codes: (060.2330) Fiber optics communications; (190.4380) Nonlinear optics, four-wave mixing; (230.7405) Wavelength conversion devices; (190.4390) Integrated optics.
\end{abstract}

\section{Introduction}

Wavelength conversion based on four wave mixing (FWM) in silicon waveguides has attracted considerable research interest due to the ultra-compactness, potential for integration with electronics, broad working bandwidth and high-speed operation of those waveguides [1-3]. In addition, the strong waveguide confinement facilitates dispersion engineering by nano-engineering the dimensions of the silicon waveguide. Broad band wavelength conversion using FWM relies on broad band phase matching, which requires careful control of the group-velocity dispersion (GVD), since in the undepleted-pump regime the bandwidth of the degenerate FWM is inversely proportional to the square root of the product of the interaction length and the GVD [4]. However, the GVD of the waveguide is very sensitive to the dimensions of the silicon waveguide, which leads to strict dimensional tolerances in the fabrication. Using higher-order waveguide dispersion to achieve phase matching in discrete bands can also enable wavelength conversion of signals with a large frequency separation. Recently, using a pulsed pump with a very high peak power, a mid-infrared optical signal was parametrically amplified and wavelength converted into the telecom L band [5].

In this paper, wavelength conversion across six telecommunication bands (O- E- S- C- L- and U- bands) from $1258 \mathrm{~nm}$ to $1726 \mathrm{~nm}$ is demonstrated using discrete FWM in a silicon waveguide with a continuous wave (CW) pump with a launched power of only $14.5 \mathrm{dBm}$.

\section{Phase matching in discrete bands using higher order dispersion}

In a FWM process, phase matching between the pump, signal and idler occurs the pump frequency provided nonlinear phase mismatch due to Kerr nonlinearity can be ignored, which is the case at moderate pump power levels, and dispersion terms higher than $\beta_{3}$ can be neglected. Here and in the following $\beta_{i}$ denotes the $\mathrm{i}^{\text {th }}$ order derivative of the propagation constant $\beta$ with respect to angular frequency. Depending on the value of $\beta_{2}$, this usually results in a continuous wavelength conversion bandwidth centered around the pump frequency. However, considering higher dispersion orders up to $\beta_{5}$ only are significant, the linear phase matching condition becomes

$$
\Delta \beta=\frac{1}{12} \beta_{4} \Delta \omega^{4}+\beta_{2} \Delta \omega^{2}=0
$$

where $\Delta \omega=\omega_{s}-\omega_{p}$ is the angular frequency separation between the signal and pump. If $\beta_{2} \beta_{4}<0$, phase matching also occurs for $\Delta \omega= \pm \sqrt{-12 \beta_{2} / \beta_{4}}$, which gives rise to discrete wavelength conversion bands on top of the main (centre) conversion bandwidth.
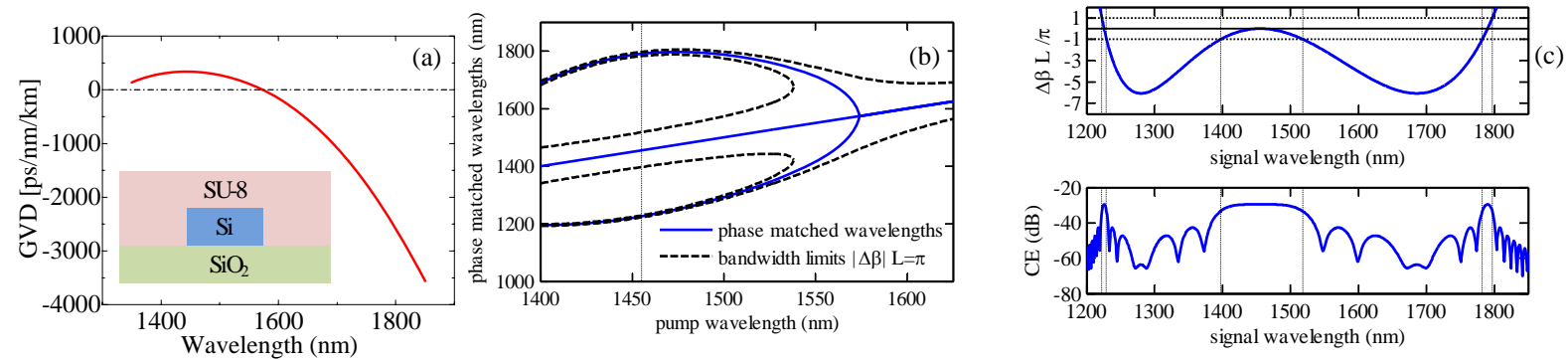

Fig. 1. (a) Simulated GVD for the silicon waveguide with cross-sectional dimensions of $450 \times 240 \mathrm{~nm}^{2}$; (b) phase matched wavelengths and corresponding conversion band limits as a function of pump wavelength; (c) phase mismatch as a function of signal wavelength and predicted conversion efficiency (CE) for a pump wavelength of $1455 \mathrm{~nm}$. 
Fig. 1a shows the GVD simulated using a custom-made finite-difference mode solver for the transverse-electric (TE) mode of a silicon waveguide with cross-sectional dimensions of $450 \times 240 \mathrm{~nm}^{2}$. For this waveguide, the condition $\beta_{2} \beta_{4}<0$ for which discrete band phase matching occurs is satisfied for pump wavelengths up to $1574 \mathrm{~nm}$. In practice, however, even dispersion orders higher than $\beta_{4}$ need to be considered for ultra-wideband processes and equation (1) needs to be modified accordingly. The phase matching diagram (Fig. 1b) represents the values of the phase matched wavelengths as a function of pump wavelength, assuming no nonlinear phase mismatch contribution and dispersion orders up to $\beta_{14}$. The diagram also shows the wavelengths for which $|\Delta \beta \cdot L|=\pi$, which are a good approximation of the limits of the wavelength conversion bands. On top of a continuous conversion band around the pump wavelength, two discrete conversion bands appear up to a pump wavelength of about $1535 \mathrm{~nm}$, over which they merge with the centre continuous conversion band. It should be pointed out that the exact values of the phase matched wavelengths represented in Fig. 1b depend on the accuracy of numerical prediction of the dispersion profile, which is itself strongly dependent on the exact dimensions of the waveguide. Fig. 1c also shows the phase mismatch factor and the numerically predicted conversion bands when the waveguide is pumped at $1455 \mathrm{~nm}$.

\section{Experiment and results}

The silicon nanowire used in the experiment is a dispersion engineered straight 3.6-mm long silicon waveguide, which includes tapering sections for low-loss interfacing with optical fiber. The main waveguide section is $\sim 3 \mathrm{~mm}$ long and has a cross section of $450 \times 240 \mathrm{~nm}^{2}$ while the tapering sections are $\sim 0.3 \mathrm{~mm}$ long each and their width is inversely tapered from $450 \mathrm{~nm}$ to $20 \mathrm{~nm}$ [6]. This tapering enables efficient coupling between the silicon waveguide and the larger polymer waveguide (SU8-2005) into which the whole silicon structure is embedded. The device has a silicon-on-insulator (SOI) structure, with the silicon waveguide placed on a $\mathrm{SiO}_{2} / \mathrm{Si}$ substrate. At $1550 \mathrm{~nm}$, the measured propagation loss is $4.3 \mathrm{~dB} / \mathrm{cm}$ and the fiber coupling loss is $1.5 \mathrm{~dB}$ per facet while at $1250 \mathrm{~nm}$ those values are $3 \mathrm{~dB} / \mathrm{cm}$ and $4.3 \mathrm{~dB}$ per facet, respectively.

The CW light at $1455 \mathrm{~nm}$ is generated by a Raman laser source, acting as the pump of the FWM process. Three tunable CW lasers spanning the O- E- S- and C-bands $(1250 \mathrm{~nm}-1565 \mathrm{~nm})$ are used as the signal source. The pump and signal are coupled together using a 3-dB coupler and then launched into the silicon nanowire. Both the pump and signal are aligned to the TE polarization. The launched pump power is $14.5 \mathrm{dBm}$. The conversion efficiency is measured as a function of the signal wavelength, as shown in Fig. 2a. We can see the main conversion bandwidth of $100 \mathrm{~nm}$ around the pump wavelength and the discrete conversion bandwidth of $10 \mathrm{~nm}$ around $1258 \mathrm{~nm}$. Fig. $2 \mathrm{~b}$ and 2c show the input and output spectra with the CW pump at $1455 \mathrm{~nm}$ and the CW signal at $1258 \mathrm{~nm}$, respectively. A conversion efficiency of $-36 \mathrm{~dB}$ is obtained for the wavelength conversion from $1258 \mathrm{~nm}$ to $1726 \mathrm{~nm}$. In addition, a peak from the Raman Stokes-shifted pump is observed at $1575 \mathrm{~nm}$.
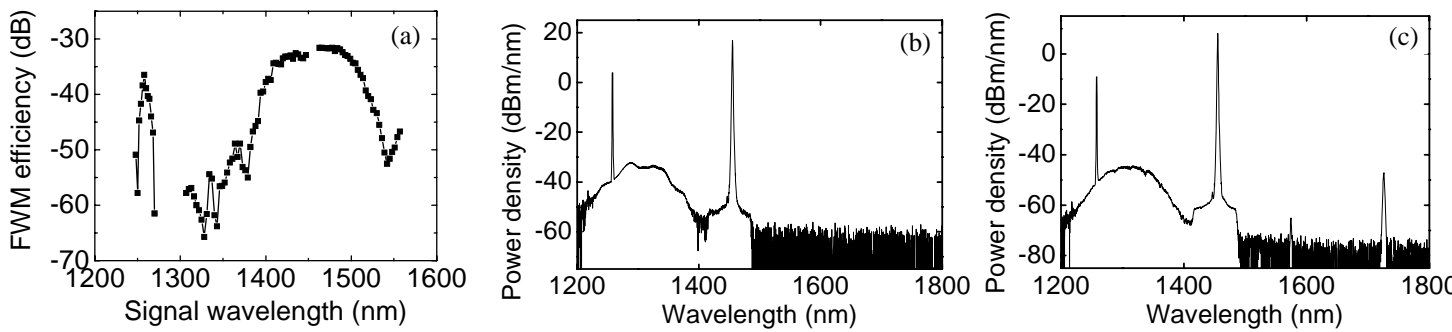

Fig. 2. (a) Measured FWM efficiency as a function of signal wavelength for the pump at $1455 \mathrm{~nm}$; (b) and (c) spectra at the input and output of the silicon nanowire with the CW pump at $1455 \mathrm{~nm}$ and the CW signal at $1258 \mathrm{~nm}$.

\section{Conclusions}

We have demonstrated 468-nm wavelength conversion based on phase matching in discrete bands using higher order dispersion in a silicon nanowire. CW light at $1258 \mathrm{~nm}$ is converted to $1726 \mathrm{~nm}$ using FWM between discrete bands with a CW pump at $1455 \mathrm{~nm}$. These results show the feasibility of inter-band wavelength conversion between different telecommunication bands.

\section{References}

[1] H. Hu, et al., "Ultra-high-speed wavelength conversion in a silicon photonic chip,” Opt. Express 19, 19886 (2011).

[2] A. C. Turner-Foster, et al., "Frequency conversion over two-thirds of an octave in silicon nanowaveguides,” Opt. Express 18, 1904 (2010).

[3] Q. Lin, et al., "Ultrabroadband parametric generation and wavelength conversion in silicon waveguides,” Opt. Express 14, 4786 (2006).

[4] M. A. Foster, et al., "Broad-band continuous-wave parametric wavelength conversion in silicon nanowaveguides,” Opt. Express 15, 12949 (2007).

[5] B. Kuyken, et al.,"Frequency conversion of mid-infrared optical signals into the telecom band using nonlinear silicon nanophotonic wires," OFC 2011, paper OThU4.

[6] M. Pu, et al., "Ultra-low-loss inverted taper coupler for silicon-on-insulator ridge waveguide,” Opt. Commun. 283, 3678 (2010) 